\title{
Femoral Facial Syndrome Associated with a De Novo Complex Chromosome 2q37 Rearrangement
}

\section{Malte Spielmann, ${ }^{1,2,3}$ Sylvie Marx, ${ }^{4}$ Gotthold Barbi, ${ }^{5}$ Ricarda Flöttmann, ${ }^{1}$ Hildegard Kehrer-Sawatzki, ${ }^{5}$ Rainer König, ${ }^{6}$ Denise Horn, ${ }^{1}$ Stefan Mundlos, ${ }^{1,2,3}$ Sean Nader, ${ }^{4}$ and Guntram Borck ${ }^{5 *}$}

\author{
${ }^{1}$ Institute for Medical and Human Genetics, Charité-Universitätsmedizin Berlin, Berlin, Germany \\ ${ }^{2}$ Max Planck Institute for Molecular Genetics, Berlin, Germany \\ ${ }^{3}$ Berlin-Brandenburg Center for Regenerative Therapies (BCRT), Berlin, Germany \\ ${ }^{4}$ Department of Pediatric Orthopedics, Schön Klinik Vogtareuth, Vogtareuth, Germany \\ ${ }^{5}$ Institute of Human Genetics, University of Ulm, Ulm, Germany \\ ${ }^{6}$ Institute of Human Genetics, University of Frankfurt, Frankfurt/Main, Germany \\ Manuscript Received: 10 October 2015; Manuscript Accepted: 30 December 2015
}

The femoral facial syndrome (FFS) is a rare congenital anomaly syndrome characterized by bilateral femoral hypoplasia and facial dysmorphism. The etiology of FFS is currently unknown but maternal/gestational diabetes has been proposed as a strong risk factor for syndromic femoral hypoplasia. In affected children born to non-diabetic mothers, a genetic contribution to FFS is suspected; however, no chromosomal anomalies or gene mutations have been identified so far. Here, we report on a girl with FFS and a de novo complex chromosome rearrangement of terminal chromosome 2q37.2. Radiographs of the pelvis and lower limbs showed bilateral shortening and bowing of the femur and radiographs of hands and feet revealed a brachydactyly type E (BDE). Using high resolution array-CGH, qPCR, and FISH, we detected a $\sim 1.9 \mathrm{Mb}$ duplication in the chromosomal region $2 \mathrm{q} 37.2$ and a $\sim 5.4 \mathrm{Mb}$ deletion on chromosome $2 \mathrm{q} 37.3$ that were absent in the parents. The duplication contains six genes and the deletion encompasses 68 genes; the latter has previously been shown to cause BDE (through haploinsufficiency for HDAC4) but not femoral hypoplasia. Therefore, we propose that the duplication $2 q 37.2$ could be causative for the femur phenotype. To the best of our knowledge, our report is the first to propose a genetic cause in a case of FFS. (c) 2016 Wiley Periodicals, Inc.

Key words: femoral facial syndrome (FFS); femoral hypoplasia unusual face syndrome (FHUFS); de novo complex genomic rearrangement; array-CGH; HACNS1; limb enhancer element 921

\section{INTRODUCTION}

The femoral facial syndrome (FFS; OMIM 134780) is a rare congenital anomaly syndrome mainly characterized by bilateral femoral hypoplasia and facial dysmorphism including a short nose with a broad tip and hypoplastic alae nasi, a long philtrum, a thin
How to Cite this Article: Spielmann M, Marx S, Barbi G, Flöttmann R, Kehrer-Sawatzki H, König R, Horn D, Mundlos S, Nader S, Borck G. 2016. Femoral facial syndrome associated with a de novo complex chromosome 2q37 rearrangement.

Am J Med Genet Part A 170A:1202-1207.

upper lip, upward slanting palpebral fissures, and micrognathia [Daentl et al., 1975; Baraitser et al., 1994; Leal et al., 2003; Paladini et al., 2007; Ho et al., 2008]. Additional skeletal malformations including 11 pairs of ribs, clubfoot, and upper limb involvement, as well as cleft palate and renal anomalies have been reported. FFS - also referred to as femoral hypoplasia unusual face syndrome (FHUFS)—mostly occurs as a sporadic condition although rare observations have suggested autosomal dominant transmission [Robinow et al., 1995]. There is a strong sex bias with the vast majority of reported patients being female.

The etiology of FFS is unknown. Up to $1 / 3$ of affected individuals are infants of diabetic mothers [Johnson et al., 1983; Castori, 2013], suggesting that maternal/gestational diabetes is a strong risk factor for complex femoral hypoplasia. At least among the affected children born to non-diabetic mothers, a genetic contribution to FFS is

Conflict of interest: None.

*Correspondence to:

Guntram Borck, Institute of Human Genetics, University of Ulm, AlbertEinstein-Allee 11, 89081 Ulm, Germany.

E-mail: guntram.borck@uni-ulm.de

Article first published online in Wiley Online Library

(wileyonlinelibrary.com): 29 January 2016

DOI 10.1002/ajmg.a.37560 
suspected, but no chromosomal anomalies or gene mutations have been reported so far. Here, we report on a girl with FFS and a de novo complex chromosome rearrangement of terminal chromosome $2 \mathrm{q}$.

\section{PHENOTYPIC DATA}

The patient was seen at ages 5 weeks and 2 years in the genetic outpatient clinic because of multiple congenital anomalies, and in pediatric orthopedics for management of femoral hypoplasia. Written informed consent was obtained from the parents and DNA was extracted from blood samples of the patient and her parents.

Four additional unrelated children with a clinical diagnosis of FFS were enrolled at Schön Klinik Vogtareuth and additional eight patients with isolated or syndromic forms of femoral hypoplasia were referred to the Max-Planck-Institute for Molecular Genetics, Berlin, for the identification of the underlying cause. The affected children's mothers were non diabetic. FFS was diagnosed based on the presence of bilateral femoral hypoplasia and facial dysmorphism.

\section{Clinical Evaluation}

The proposita was the first child of a 21-year-old healthy mother and a 30 year-old healthy father who were unrelated and of German origin. At 23 weeks of gestation, a shortening of the femora was noticed on fetal ultrasound examination. There was no pre-existing maternal diabetes and no gestational diabetes. Birth was at 37 weeks of gestation by spontaneous delivery with a length of $41 \mathrm{~cm}(<3 \mathrm{rd}$ centile, $-3.5 \mathrm{SD}$ ), a weight of $2385 \mathrm{~g}$ ( $<3$ rd centile, $-1.3 \mathrm{SD}$ ), and an occipitofrontal circumference of $34 \mathrm{~cm}$ (50th centile). Physical examination showed severely shortened thighs (more pronounced on the left side) and cleft palate. She had facial dysmorphic features consisting of a prominent forehead, epicanthal folds, a short nose with anteverted nares, a long philtrum, a thin upper lip, apparently low-set ears, and micrognathia (Fig. 1A). She had a short neck, slender fingers, and a bilateral single transverse palmar crease. At the age of 4 months, the first surgery was done to repair the cleft palate. Delayed speech development and tympanic effusion necessitated several tympanostomy tube placements in early childhood. Additional examinations revealed mild aortic insufficiency, 11 pairs of ribs, and a left sided pelvic kidney. At the age of 2 years and 3 months, her height was $74 \mathrm{~cm}(<3 \mathrm{rd}$ centile, $-5.1 \mathrm{SD})$, whereas weight and OFC were 3rd-10th centile and 50th-75th centile, respectively. She started walking with the help of orthosis at 3 years. Hip surgery was performed on the less affected left side at the age of 4 years with proximal femoral valgisation osteotomy followed by hip-/knee release, and femur-lengthening with external fixation on the shorter right leg at the age of 5 years to obtain equal leg length. At that time, she did not need support for ambulation, but due to flat foot, she underwent an Evans procedure for calcaneal-lengthening at 8 years. She had some learning difficulties in mathematics but attended a regular primary school and had no intellectual disability. The clinical presentation was compatible with FFS.

Radiographs of the pelvis and lower limbs at 6 months showed shortening and bowing of the right femur and more severe shortening and proximal hypoplasia of the left femur. The proximal femoral epiphyses were unossified and the distal femoral epiphyses were abnormal. At the age of 8 years, a radiograph of the pelvis and lower limbs revealed scoliosis, asymmetric pelvis, asymmetric length of the misshapen femora, abnormal acetabulae, hip dislocation, and thin tibiae and fibulae (Fig. 1B). Only 11 rib pairs were present and she had scoliosis (Fig. 1C). Radiographs of the hands showed short metacarpalia (I, IV, and V on the left and III-V on the right, Fig. 1D), consistent with brachydactyly type E (BDE). Radiographs of the right and left foot showed short hypoplastic metatarsals II-V as well as short and hypoplastic phalangeal bones II-V (Fig. 1E), again demonstrating BDE.

\section{METHODS}

\section{Microarray-Based Comparative Genomic Hybridization (Array CGH)}

Array CGH was performed using a whole genome $1 \mathrm{M}$ oligonucleotide array (Agilent, Santa Clara, CA). One molar array was analyzed by Feature Extraction v9.5.3.1 and CGH Analytics v3.4.40 software (Agilent, Santa Clara, CA). Analysis settings were as follows: aberration algorithm: ADM-2; threshold: 6.0; window size: $0.2 \mathrm{Mb}$; filter: 5 probes, $\log 2$ ratio $=0.29$. The genomic profile was visualized by the CytoGenomics Software (Agilent CytoGenomics. 2.0., Agilent Technologies, Inc.) and SignalMap software (v1.9.0.03, NimbleGen). Data were submitted to the DECIPHER database (http://decipher.sanger.ac.uk) [Firth et al., 2009]; accession number: patient 314714.

\section{Quantitative Real-Time PCR [qPCR]}

qPCR was performed as described previously [Klopocki et al., 2008], using genomic DNA of the patient and her parents to confirm the deletion and duplication and to test for de novo occurrence. Primer sequences for $\mathrm{qPCR}$ are $2 \mathrm{q} 37.2 \_\mathrm{N}$ $\left(5^{\prime}\right.$-TCTACTTCCAAGGTGACGCC- $\left.3^{\prime}\right)$ as control, 2q37.2_O (5'-TGCTCACTGACCTCTGTTGG-3'), 2q37.2_P (5'-TAGTGTGACAGCTGGCGTTC- $\left.3^{\prime}\right)$, 2q27.2_Q (5' CAGTCG-3 $\left.3^{\prime}\right)$ for the duplication, 2q37.2_R (5'-TATAATTCCGC CACCCAAAG-3') and 2q37.2_S (5'-AGATGGAGCAGAGAGGACGA-3') as control, 2q37.2_T (5'-GCTCTGGCCCAAGTTTAGTG-3'), qHDAC4_ex12 (5'-CGGTCACCTGTCTGAAGAGC $\left.-3^{\prime}\right)$, 2q37.2_N (5'-CTTCGAGATGTGCCCACTTT-3') for the deletion, and 2q37.2_U (5'-AGAGAGCGAGAGGCAGTTTG-3') as another control.

\section{Fluorescence In Situ Hybridisation (FISH)}

G-banded chromosome analysis and FISH were performed according to standard procedures, and for FISH a 2q subtelomeric probe (ToTelVysion-Kit, Abbott; 2QTEL47 Locus) and a whole chromosome paint specific for chromosome 2 (WCP2, Vysis) were used.

\section{RESULTS \\ Cytogenetic and Molecular Genetic Analyses}

The patient had a normal G-banded karyotype (46,XX). High resolution array-CGH detected $\mathrm{a} \sim 1.9 \mathrm{Mb}$ duplication in the 

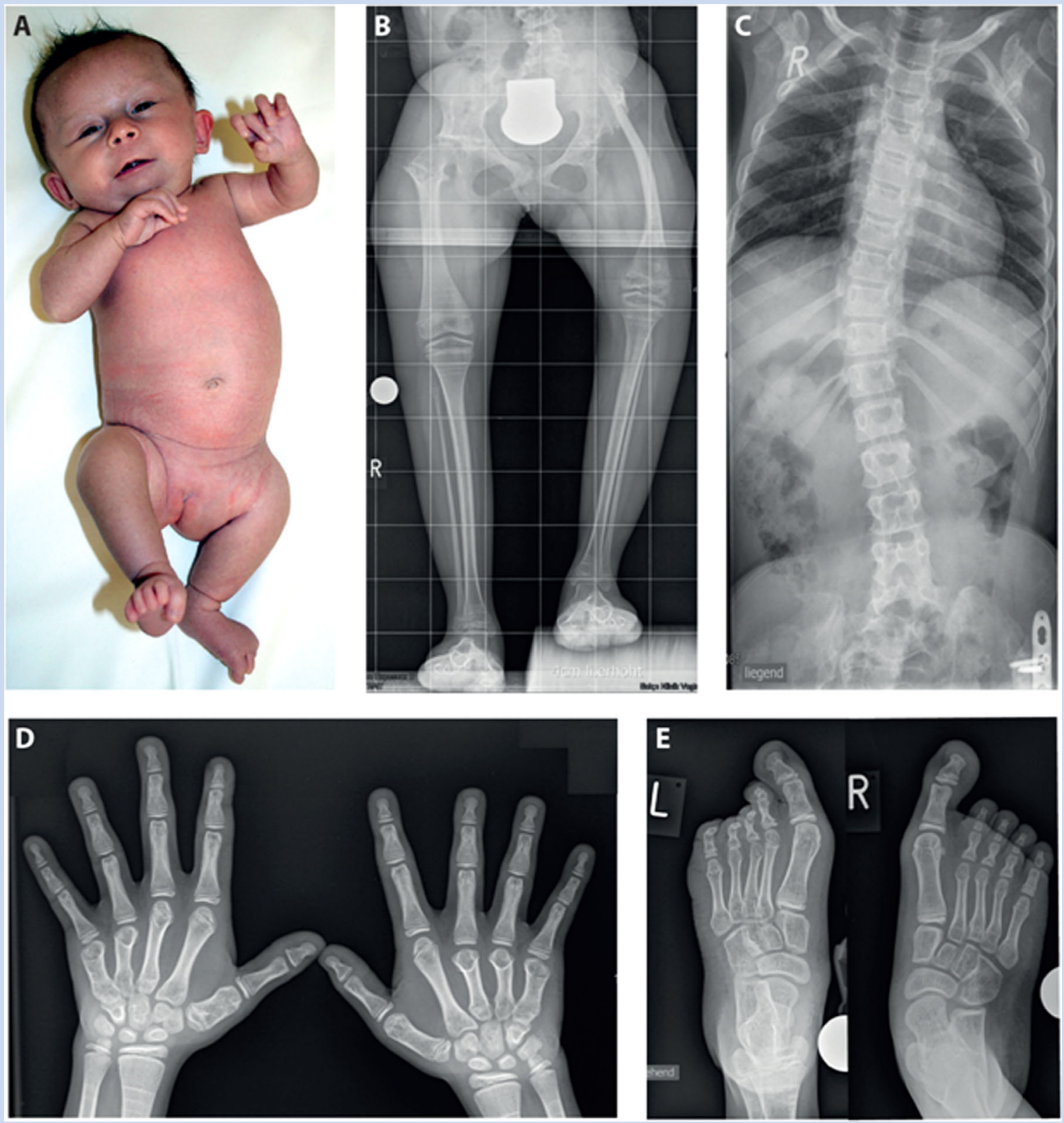

FIG. 1. (A) Clinical picture of the affected individual at the age of 6 months: note shortening and bowing of the right femur and more severe shortening and proximal hypoplasia of the left femur. (B) At the age of 8 years, radiograph of the pelvis and lower limbs shows scoliosis, asymmetric pelvis, asymmetric length of the misshapen femora, abnormal acetabulae, hip dislocation, and thin tibiae and fibulae. (C) Eleven rib pairs and scoliosis. (D) Radiographs of the right and left hands showing a brachydactyly type E (BDE) with short hypoplastic metacarpals III-V. (E) Radiographs of the right and left foot showing short hypoplastic metatarsals II-V [corresponding to BDE] as well as short and hypoplastic phalangeal bones II-V. [Color figure can be seen in the online version of this article, available at http://wileyonlinelibrary.com/journal/ajmga].

chromosomal region 2q37.2 (hg19 chr2:235,670,827-237,590,836) and a $\sim 5.4 \mathrm{Mb}$ deletion on chromosome 2q37.3 (hg19 chr2:237,605,322-243,041,364) (Fig. 2A). qPCR confirmed both the duplication and the deletion in the patient and showed their absence in the parents, consistent with a de novo occurrence of this complex rearrangement (data not shown). FISH using a probe mapping to the deletion region again confirmed the deletion in the patient (Fig. 2B). FISH on parental metaphase chromosomes showed a signal on each chromosome 2 homolog, ruling out a balanced translocation (data not shown). Finally, FISH with a whole-chromosome paint specific for chromosome 2 painted the two chromosome 2 homologs in the patient and did not show 

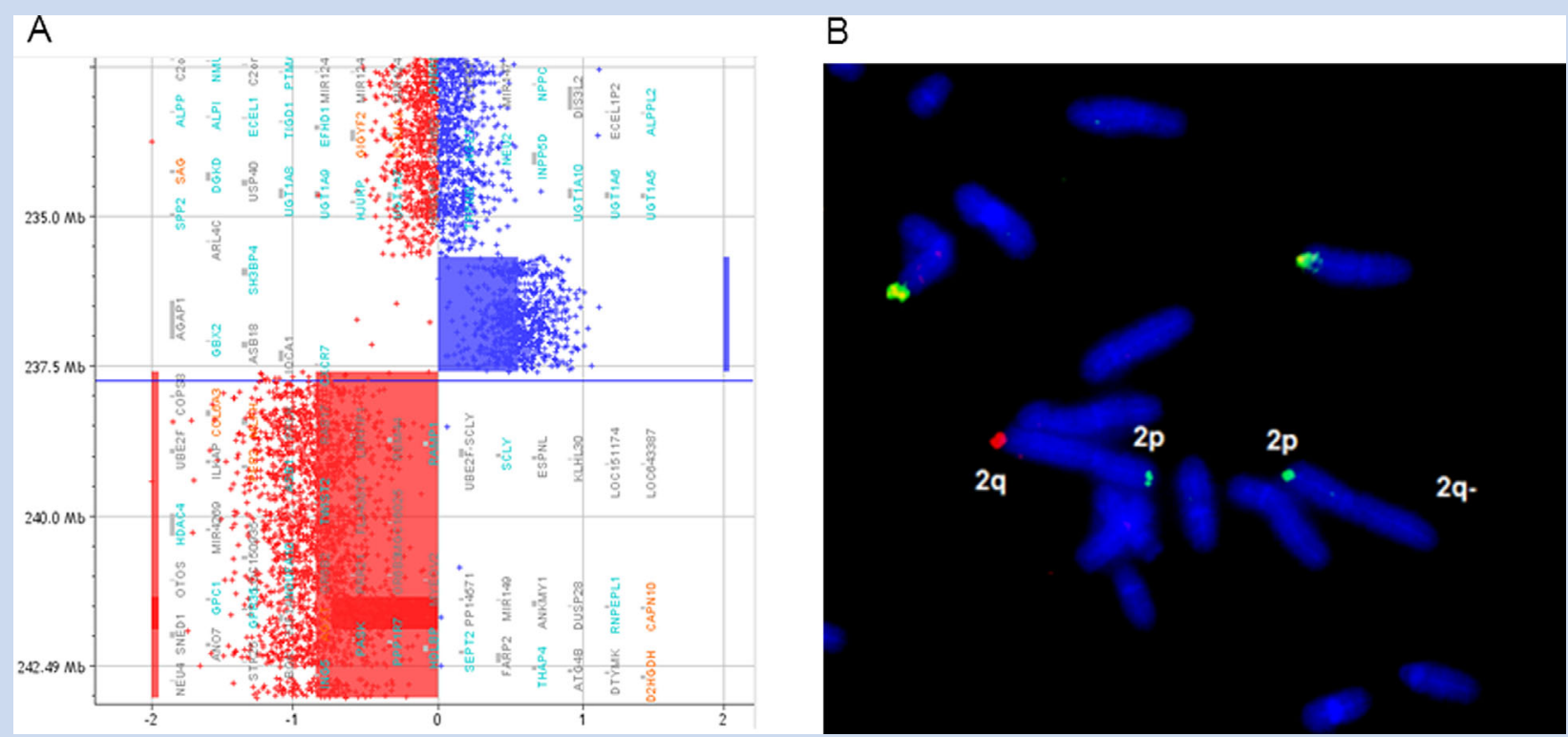

FIG. 2. (A) High resolution array-CGH detected a $\sim 1.9 \mathrm{Mb}$ duplication in the chromosomal region $2 q 37.2$ (blue) and a $\sim 5.4 \mathrm{Mb}$ deletion on chromosome 2q37.3 (red). (B) FISH with subtelomere-specific chromosome 2 probes confirms a deletion 2 qter on one chromosome. [Color figure can be seen in the online version of this article, available at http://wileyonlinelibrary.com/journal/ajmga].

signals on other chromosomes (data not shown). The duplication contains six genes: SH3BP4, AGAP1, GBX2, ASB18, IQCA1, and CXCR7, and the deletion encompasses 68 genes (Fig. 3). In 12 additional unrelated children with femoral hypoplasia or a clinical diagnosis of FFS, we detected no chromosome $2 \mathrm{q} 37$ imbalance and no potentially causative copy number variants genome-wide using high-resolution array CGH.

\section{DISCUSSION}

The FFS was first described by Daentl et al. [1975] and its etiology has remained elusive since then. This might be due to the fact that FFS is very rare, occurs sporadically, and most likely does not represent a single homogeneous genetic entity. While some authors have called into question the existence of FFS as a separate entity, the characteristic facial features in combination with bilateral femoral hypoplasia argue in favor of a possibly genetic entity, at least in the absence of maternal diabetes [Lord and Beighton, 1981]. To the best of our knowledge, our report is the first to propose a genetic cause in a case of FFS. Using a combination of array CGH, qPCR, and FISH, we have identified and characterized a de novo deletion/duplication together encompassing more than 70 genes in terminal 2q. Since both, the deletion as well as the duplication occurred de novo, we conclude that they resulted from the same mutational event. A replication-based mechanism involving multiple templates switches such as fork stalling and template switching (FoSTeS) or microhomology-mediated break-induced replication (MMBIR) may have caused this complex genomic rearrangement (CGR). The size, gene content, and de novo occurrence of this CGR argue in favor of its pathogenicity. Absence of 2qter anomalies in four additional patients with FFS strengthens the clinical impression that FFS may be genetically heterogeneous, although we cannot exclude the possibility that a deletion or duplication below the resolution of high resolution array CGH (resolution $<20 \mathrm{~kb}$ ) or a mutation of a gene mapping to the deletion or duplication interval is present in other patients with FFS.

Terminal deletions of chromosome $2 \mathrm{q}$ of various lengths cause the $2 q 37$ deletion syndrome that includes mild-moderate intellectual disability; facial dysmorphisms; behavioral manifestations including autism spectrum disorders; and central nervous system, ocular, cardiac, gastrointestinal, and genitourinary anomalies in nearly one-third of patients [Falk and Casas, 2007]. Deletions involving the subtelomeric band 2q37.3 and heterozygous mutations of the histone deacetylase encoding HDAC4 gene cause brachydactyly mental retardation syndrome (BDMR) [Williams et al., 2010; Leroy et al., 2013], which is characterized by developmental delay, intellectual disability and BDE [Williams et al., 2010; Leroy et al., 2013]. Intellectual disability is not mandatory in HDAC4 haploinsufficiency, and BDE in our patient can be explained by HDAC4 deletion [Wheeler et al., 2014; Jean-Marcais et al., 2015]. Femoral hypoplasia is not part of the classical 2q37 deletion syndrome or BDMR, indicating that haploinsufficiency for one of the deleted genes is an unlikely pathomechanisms. However, we note that asymmetrical length of legs has been described in two patients carrying 2q37.3 deletions and there is some phenotypic overlap of the patient presented here with both: the Femoral Facial Syndrome and the 2q37.3 deletion syndrome (Table I) [Leroy et al., 2013]. While we cannot formally exclude that the chromosome 2 rearrangement and severe femoral hypoplasia co-occur by chance, it is conceivable that the duplication of one of 


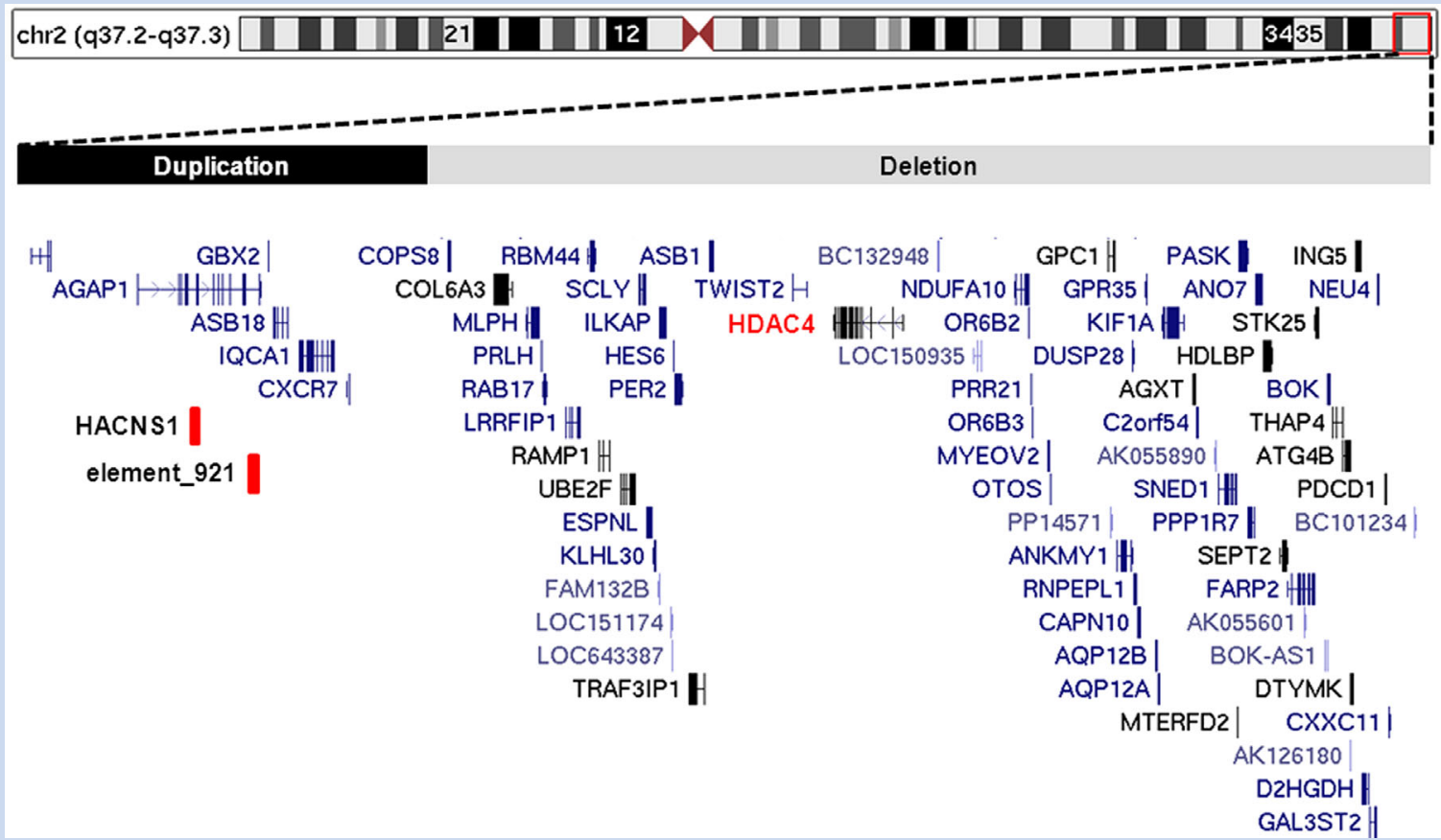

FIG. 3. Femoral facial syndrome associated with a de novo complex chromosome $2 q 37$ rearrangement. The terminal deletion $2 q 37.3$ is known to associate with brachydactyly type $\mathrm{E}$ and mental retardation through haploinsufficency for HDAC4 (highlighted in red). Two cis-regulatory limb enhancer elements are located in the duplicated region: HACNS1 and the enhancer element 921 . Their duplication might contribute to the femoral hypoplasia phenotype. [Color figure can be seen in the online version of this article, available at http://wileyonlinelibrary.com/journal/ajmga].

TABLE I. Facial and Skeletal Characteristics of Deletion 2q37.3, Femoral-Facial Syndrome and Presently Reported Patient

Facial dysmorphism

2q37.3 deletion syndrome* OMIM 600430

Prominent forehead

Epicanthal folds

Short nose

V-shape of the nasal tip

Anteverted nares

Long philtrum

Thin upper vermillion

Low-set ears

Micrognathia

Cleft palate

Skeletal abnormalities

Brachydactyly Type E

Short metacarpals

Short metatarsals

Short phalanges

Shortened, bowed femur

Scoliosis

Asymmetric pelvis

Abnormal acetabulae

Hip dislocation

Thin tibiae and fibulae

$+$

$+$

$+$

$-$

$+$

$+$

$+$

$+$

(+) $2 / 14$

$-$

$-$

\section{Femoral facial syndrome}

OMIM 134780

Patient

+12/14 Leroy et al. [2013]

$(+)(5) / 14$ Leroy et al. [2013]

- +

- + 
the six genes in 2q37.2-q37.3 results in femoral hypoplasia or that a more complex mechanism plays a role, e.g., coinheritance of a rare variant on the non-rearranged allele. While we cannot formally exclude that the chromosome 2 rearrangement and severe femoral hypoplasia co-occur by chance, it is conceivable that the duplication of one of the six genes in 2q37.2-q37.3 results in femoral hypoplasia or that a more complex mechanism plays a role, e.g., coinheritance of a rare variant on the non-rearranged allele. Of the six duplicated genes, only the transcription factor GBX2 (Gastrulation Brain Homeobox 2) is known to be expressed in the developing limb [Niss and Leutz, 1998]. Gbx2 null mice do not show limb anomalies but the duplication of the $G b \times 2$ might contribute to the phenotype of the patient [Wassarman et al., 1997].

It is notable that at least two cis-regulatory elements are located in the duplicated region: the human-accelerated conserved noncoding sequence 1 (HACNS1) is located in an intron of AGAP1 (also known as CENTG2) which encodes a GTPase activating protein involved in the regulation of endosome function; and limb enhancer element_921 (Fig. 3) [Visel et al., 2007; Prabhakar et al., 2008]. In transgenic studies, it was previously shown that HACNS1 acts a key limb enhancer element that may contribute to the uniquely human aspects of digit and limb patterning [Prabhakar et al., 2008]. Thus, duplication of the limb enhancer HACNS1 is a candidate contributor to the femoral hypoplasia phenotype of the patient reported here. Similar duplications of cis-regulatory elements such as the SHH limb enhancer element ZRS (Zone of Regulatory Sequence) have been shown to cause a variety of limb phenotypes ranging from triphalangeal thumb to cup shaped polysyndactyly [Spielmann and Klopocki, 2013; Lohan et al., 2014]. In conclusion and not withstanding the unknown pathomechanisms, to our knowledge, our report is the first to describe a genetic cause of FFS and may thus help elucidating the genetic determinants of femoral growth.

\section{ACKNOWLEDGMENT}

We thank the family for participation in the study.

\section{REFERENCES}

Baraitser M, Reardon W, Oley C, Fixsen J. 1994. Femoral hypoplasia unusual facies syndrome with preaxial polydactyly. Clin Dysmorphol 3:40-45.

Castori M. 2013. Diabetic embryopathy: A developmental perspective from fertilization to adulthood. Mol Syndromol 4:74-86.

Daentl DL, Smith DW, Scott CI, Hall BD, Gooding CA. 1975. Femoral hypoplasia-unusual facies syndrome. J Pediatr 86:107-111.

Falk RE, Casas KA. 2007. Chromosome 2q37 deletion: Clinical and molecular aspects. Am J Med Genet C Semin Med Genet 145C:357-371.

Firth HV, Richards SM, Bevan AP, Clayton S, Corpas M, Rajan D, Van Vooren S, Moreau Y, Pettett RM, Carter NP. 2009. DECIPHER: Database of chromosomal imbalance and phenotype in humans using ensembl resources. Am J Hum Genet 84:524-533.

Ho AL, Lefloch N, Levy ML, Bird LM. 2008. Femoral facial syndrome: A case report with coexistent hydrocephaly. Clin Dysmorphol 17:259-263.
Jean-Marcais N, Decamp M, Gerard M, Ribault V, Andrieux J, Kottler ML, Plessis G. 2015. The first familial case of inherited 2q37.3 interstitial deletion with isolated skeletal abnormalities including brachydactyly type E and short stature. Am J Med Genet A 167A:185-189.

Johnson JP, Carey JC, Gooch WM 3rd, Petersen J, Beattie JF. 1983. Femoral hypoplasia-unusual facies syndrome in infants of diabetic mothers. J Pediatr 102:866-872.

Klopocki E, Ott CE, Benatar N, Ullmann R, Mundlos S, Lehmann K. 2008. A microduplication of the long range $\mathrm{SHH}$ limb regulator (ZRS) is associated with triphalangeal thumb-polysyndactyly syndrome. J Med Genet 45:370-375.

Leal E, Macias-Gomez N, Rodriguez L, Mercado FM, Barros-Nunez P. 2003. Femoral-facial syndrome with malformations in the central nervous system. Clin Imaging 27:23-26.

Leroy C, Landais E, Briault S, David A, Tassy O, Gruchy N, Delobel B, Gregoire MJ, Leheup B, Taine L, Lacombe D, Delrue MA, Toutain A, Paubel A, Mugneret F, Thauvin-Robinet C, Arpin S, Le Caignec C, Jonveaux P, Beri M, Leporrier N, Motte J, Fiquet C, Brichet O, MozelleNivoix M, Sabouraud P, Golovkine N, Bednarek N, Gaillard D, DocoFenzy M. 2013. The 2q37-deletion syndrome: An update of the clinical spectrum including overweight, brachydactyly, and behavioral features in 14 new patients. Eur J Human Genet 21:602-612.

Lohan S, Spielmann M, Doelken SC, Flottmann R, Muhammad F, Baig SM, Wajid M, Hulsemann W, Habenicht R, Kjaer KW, Patil SJ, Girisha KM, Abarca-Barriga HH, Mundlos S, Klopocki E. 2014. Microduplications encompassing the Sonic hedgehog limb enhancer ZRS are associated with Haas-type polysyndactyly and Laurin-Sandrow syndrome. Clin Genet 86:318-325.

Lord J, Beighton P. 1981. The femoral hypoplasia-unusual facies syndrome: A genetic entity? Clin Genet 20:267-275.

Niss K, Leutz A. 1998. Expression of the homeobox gene GBX2 during chicken development. Mech Dev 76:151-155.

Paladini D, Maruotti GM, Sglavo G, Penner I, Leone F, D'Armiento MR, Martinelli P. 2007. Diagnosis of femoral hypoplasia-unusual facies syndrome in the fetus. Ultrasound Obstet Gynecol 30:354-358.

Prabhakar S, Visel A, Akiyama JA, Shoukry M, Lewis KD, Holt A, PlajzerFrick I, Morrison H, Fitzpatrick DR, Afzal V, Pennacchio LA, Rubin EM, Noonan JP. 2008. Human-specific gain of function in a developmental enhancer. Science 321:1346-1350.

Robinow M, Sonek J, Buttino L, Veghte A. 1995. Femoral-facial syndrome-prenatal diagnosis-autosomal dominant inheritance. Am J Med Genet A 57:397-399.

Spielmann M, Klopocki E. 2013. CNVs of noncoding cis-regulatory elements in human disease. Curr Opin Genetics Dev 23:249-256.

Visel A, Minovitsky S, Dubchak I, Pennacchio LA. 2007. VISTA Enhancer Browser-A database of tissue-specific human enhancers. Nucleic Acids Res 35:D88-D92.

Wassarman KM, Lewandoski M, Campbell K, Joyner AL, Rubenstein JL, Martinez S, Martin GR. 1997. Specification of the anterior hindbrain and establishment of a normal mid/hindbrain organizer is dependent on Gbx2 gene function. Development 124:2923-2934.

Wheeler PG, Huang D, Dai Z. 2014. Haploinsufficiency of HDAC4 does not cause intellectual disability in all affected individuals. Am J Med Genet A 164A:1826-1829.

Williams SR, Aldred MA, Der Kaloustian VM, Halal F, Gowans G, McLeod DR, Zondag S, Toriello HV, Magenis RE, Elsea SH. 2010. Haploinsufficiency of HDAC4 causes brachydactyly mental retardation syndrome, with brachydactyly type E, developmental delays, and behavioral problems. Am J Hum Genet 87:219-228. 